\title{
Colaboración científica en artículos de revistas biomédicas peruanas
}

\section{Scientific collaboration in Peruvian biomedical journals}

\author{
Charles Huamaní ${ }^{1,2}$, José Pacheco-Romero ${ }^{1}$ \\ Facultad de Medicina Humana, Universidad Nacional Mayor de San Marcos. Lima, Perú. \\ ${ }^{2}$ Instituto Nacional de Salud. Lima, Perú.
}

\begin{abstract}
Resumen
Introducción: En el área médica, los productos de las actividades de investigación se manifiestan principalmente en revistas cientificas; estos productos son cuantificables y pueden ser registrados en bases de datos especializadas. Objetivos: Realizar un análisis de los investigadores e instituciones investigadoras peruanas, determinando las características de su sociabilización e interacción, de manera de identificar los posibles nudos críticos en los que una intervención mediática pudiera ayudar a mejorar e incrementar la producción científica en medicina. Diseño: Estudio bibliométrico. Lugar: Facultad de Medicina, Universidad Nacional Mayor de San Marcos, Lima, Perú. Material bibliográfico: Artículos científicos publicados durante el periodo 2005-2008, en la base de datos SciELO Perú. Intervenciones: Se seleccionó solo los artículos científicos, identificando la autoría y filiación institucional de cada uno, confeccionando una base de datos, en la que se procedió al análisis de colaboración entre autores e instituciones. Principales medidas de resultados: Índice de firmas por autor, frecuencias, porcentajes y redes de colaboración. Resultados: Se analizó 493 artículos que reunieron 2131 firmas de 1540 autores y 271 instituciones; 56,2\% fue realizado de manera conjunta por dos instituciones. El 70,5\% de los investigadores tuvo solo un artículo registrado. Las instituciones más productivas fueron la UN Mayor de San Marcos y la U Peruana Cayetano Heredia, con 138 y 125 artículos, respectivamente. Conclusiones: La investigación médica nacional se desarrolla a través de pocos autores constantes y pocos núcleos de investigación, principalmente universitarios, que están centralizados, y con una apertura moderada a otros nodos en desarrollo.
\end{abstract}

Palabras clave (DeCS): Bibliometria; indicador de colaboración; publicaciones seriadas; gestión de ciencia, tecnología e innovación en salud; Perú.

\begin{abstract}
Background: Products of research activities in medicine are mainly expressed in scientific journals; these products are subject to quantification and may be registered in specialized databases. Objectives: To determine socialization and interaction of Peruvian researchers and research institutions in order to identify possible critical knots where media intervention might help improving and increasing scientific production in medicine. Design: Bibliometrical study. Institution: Faculty of Medicine, Universidad Nacional Mayor de San Marcos, Lima, Peru. Bibliographic material: Scientific papers published during 2005-2008 in SciELO Peru database. Interventions: Only scientific papers were selected, identifying authorship and institutional affiliation. Authorship and institutional collaboration were analyzed. Main outcome measures: Authors' signatures index, frequencies, percentages and collaboration nets. Results: Four hundred and ninety three papers were analyzed comprising 2131 signatures of 1540 authors and 271 institutions; $56,2 \%$ were elaborated jointly by two institutions; $70,5 \%$ of researchers had only one paper registered. Most productive institutions were Universidad Nacional Mayor de San Marcos and Universidad Peruana Cayetano Heredia, with 138 and 125 articles respectively. Conclusions: National medical research is accomplished by few persevering researchers and few research nuclei mainly from universities that appear centralized and with moderate aperture to other developing knots.
\end{abstract}

Key words (DeCS): Bibliometrics; collaboration indicator; serial publications; science administration, health technology and innovation; Peru.

An Fac med. 2011;72(4):261-8

\section{INTRODUCCIÓN}

En el área médica, los productos de las actividades de investigación se manifiestan principalmente en revistas científicas, libros especializados y patentes. Estos productos son cuantificables y pueden ser registrados en bases de datos especializadas, siendo la más reconocida internacionalmente el Science
Citation Index generado por el Institute for Scientific Information de Thompson Reuters (ISI-TR) ${ }^{(1)}$, que permite un análisis de la producción incluso a nivel de países.

El análisis de la bibliografía científica (bibliometría), tal como lo señalaba Garfield, creador de esta ciencia, tiene como principales limitaciones la selección tanto de las revistas como de los artículos, porque al incluir revistas indizadas, existe una exclusión de literatura sea por idioma o difusión (en el caso de ISI-TR incluye preferentemente revistas de habla inglesa), o al incluir artículos con una estructura común puede no considerar artículos de similar relevancia, pero con una estructura distinta (como los ensayos en historia de la medicina). 
Además, con frecuencia se confunde y se usa sus indicadores como criterios de importancia o calidad, cuando solo deben permitir una evaluación de los fenómenos sociales en la investigación ${ }^{(2)}$; por ello, repetidas veces se ha señalado que estos indicadores deben ser usados junto con otros indicadores.

Si bien Perú contribuye con aproximadamente 100 publicaciones al año en revistas de alto impacto ${ }^{(3)}$, no se tiene en cuenta la producción de revistas no indizadas a ISI-TR, a pesar de que puedan incluir artículos de elevada calidad. Esta 'otra' producción científica es difícil de rastrear, debido a dificultades en los mismos indizadores (por ejemplo, Medline solo utiliza la nacionalidad del autor principal del artículo, sin considerar que dentro del equipo de investigación puede haber un peruano) o a que parte de la producción peruana se publica en revistas efímeras, sin registros ni indizaciones, siendo literatura que no tendrá mayor repercusión debido a su visibilidad nula.

Teniendo presente esas consideraciones, la bibliometría ofrece una visión comparativa de la actividad científica, así como de su difusión y tendencias en la investigación. Un análisis crítico y periódico de la producción científica ayuda en la valoración y gerencia de las instituciones dedicadas a la investigación; tal es así que parte del éxito logrado en la mejora del desarrollo en investigación en países como España se debe, además de la inversión económica y dedicación, a la constante evaluación y mejoramiento de sus procesos, secundarios a los análisis periódicos sobre su investigación científica ${ }^{(4-7)}$.

Para lograr una visión integral del contexto de la investigación, el análisis suele enfocarse desde diversos puntos, tratando desde el uso de indicadores de producción -tales como cantidad de artículos por revista, autor, institución o año de publicación- y analizar con ello la 'sociología' de la investigación; también, el contenido de la información (metodología aplicada, diseño, área de investigación, estadística, entre otros) es un indicador que nos puede dar una idea de la calidad de la investigación realizada, o analizar el consumo de la información, complementando el panorama descrito ${ }^{(8)}$.

Esta investigación tiene como objetivo realizar un análisis de los investigadores e instituciones investigadoras peruanas, buscando determinar las características de su sociabilización e interacción para identificar los posibles nudos críticos en los que una intervención mediática pueda ayudar a mejorar e incrementar la producción científica en medicina. Este análisis y su consecuente autocrítica y discusión permitirán establecer el escenario de la investigación científica biomédica peruana y así realizar propuestas en la mejora de la educación médica, gerencia institucional y desarrollo científico.

\section{MÉTODOS}

Se identificó los artículos científicos publicados durante el periodo 2005 a 2008. La selección de las revistas se basó en un estudio previo en el que se confeccionó un listado de revistas dedicadas a la medicina o alguna especialidad médica vigentes hasta $2007^{(9)}$, seleccionando las indizadas a SciELO Perú, de recuperación electrónica total y actualizadas hasta 2008. Las revistas incluidas figuran en la tabla 1 .

De las revistas, seleccionamos los artículos científicos, incluyendo solo los artículos originales y comunicaciones breves que no hubieran sido publicados anteriormente y que han sido sometidos a evaluación por pares para evaluar su calidad científica (según indica la revista), y excluimos las series de casos o publicaciones que no eran investigaciones y figuraban como artículos.

No se hizo una búsqueda de publicaciones duplicadas, por lo que solo se excluyó las que indicaban haber sido publicadas previamente. Para la recolección de los datos, se accedió a la página web SciElO-Perú (www. scielo.org.pe), el Portal de Revistas Peruanas de CONCYTEC, el repositorio
SISBIB-UNMSM o la página oficial de la entidad publicadora. La totalidad de artículos incluidos se observa en la tabla 2 .

Para el análisis de la autoría y filiación institucional se confeccionó un listado de autores e instituciones y se procedió a la normalización de las diferentes variantes en la forma de escribir los nombres de un mismo autor o institución en un artículo. Para la normalización de los nombres, en los casos que era probable que el mismo autor apareciera de dos maneras distintas (igualdad en el primer apellido y la inicial del segundo apellido o del nombre), se observó la filiación institucional; en caso de coincidencia, se consideró que era el mismo autor.

Cada autor genera una firma por artículo; el número de firmas en un artículo es ilimitado; por tanto, un autor puede estar relacionado con un conjunto ilimitado de firmas, aunque estas provengan de un conjunto menor de autores. La colaboración entre los autores en las revistas se ha efectuado a partir del índice de colaboración o índice de firmas por artículo (IFA), que es el cociente del número de firmas entre el número de artículos; este indicador se utilizó para valorar de forma general a las revistas, y de forma individual a cada autor.

El número de firmas 'distintas' del total de firmas relacionadas a un autor es equivalente al número de coautores, que es un indicador cuantitativo absoluto del tamaño del grupo de trabajo de cada autor. Y las relaciones entre coautores y el número de artículos se conoce como el índice de autores por artículo, que correlaciona por tanto el tamaño del grupo de trabajo de cada autor con su productividad; los índices más elevados muestran un mayor grado de apertura para colaborar con autores diferentes.

En el caso de las instituciones, solo se ha considerado a las macroinstituciones y no a las dependencias o facultades que integra. Para valorar la productividad de una institución, se ha determinado el 
Tabla 1. Revistas médicas peruanas trimestrales incluidas en la base de datos SciELO-Perú 2007.

\begin{tabular}{ccc} 
Revista & Institución & Inicio \\
R. de Gastroenterología del Perú & S. Gastroenterología del Perú & 1981 \\
R. Peruana de Medicina Experimental y Salud Pública & Instituto Nacional de Salud & 1942 \\
Anales de la Facultad de Medicina & U. Nacional Mayor de San Marcos. F. Medicina & 1918 \\
R. Médica Herediana & U. Peruana Cayetano Heredia. F. Medicina & 1990 \\
Acta Médica Peruana & Colegio Médico del Perú & 1972 \\
R. Peruana de Pediatría & S. Peruana de Pediatría & 1927 \\
Dermatología Peruana & S. Peruana de Dermatología & 1996 \\
\hline
\end{tabular}

$\mathrm{R}=$ Revista, $\mathrm{U}=$ Universidad $\mathrm{S}=$ Sociedad, $\mathrm{F}$ = Facultad.

Modificado de: Huamani C, Pacheco-Romero J. Visibilidad y Producción de las Revistas Biomédicas Peruanas. Rev Gastroenterol Perú. $2009 ; 29(2): 132-9$

número de artículos firmados, el número de instituciones con las que ha colaborado, y el mayor número de artículos realizados con una misma institución (colaboración más intensa).

A su vez, para evaluar la colaboración institucional, se diferencia los siguientes tipos:

- Colaboración intrainstitucional: se da en los artículos de una misma macroinstitución pero que pueden ser firmados conjuntamente por diferentes departamentos, servicios o facultades.

- Colaboración interinstitucional: aquella en la que concurren al menos dos instituciones de un mismo país.
Se ha diferenciado la colaboración interinstitucional entre centros de la misma o de diferentes regiones.

- Colaboración internacional: cuando un mismo artículo lo firman instituciones de más de un país, incluyendo a Perú.

- Artículos internacionales: cuando ninguna institución es de Perú.

La colaboración científica de los autores e instituciones más productivas se valora a partir de diferentes indicadores, como a) el total de firmas de los artículos en los que han intervenido, b) el índice de firmas por artículo (IFA), c) el número de autores o instituciones con las que han colaborado, d) el nú-

Tabla 2. Artículos científicos publicados en revistas médicas peruanas e incluidas en el estudio. Periodo 2005-2008.

\begin{tabular}{c|cc|cc}
\multicolumn{1}{c|}{} & \multicolumn{2}{c}{ Artículos $(\%)^{*}$} & \multicolumn{2}{c}{ Total Publicación (\%)** } \\
RPMESP & 139 & $(28 \%)$ & 265 & $(52 \%)$ \\
AFM & 106 & $(22 \%)$ & 193 & $(55 \%)$ \\
RGP & 74 & $(15 \%)$ & 195 & $(38 \%)$ \\
RMH & 72 & $(15 \%)$ & 156 & $(46 \%)$ \\
AMP & 42 & $(9 \%)$ & 159 & $(26 \%)$ \\
RPP & 35 & $(7 \%)$ & 139 & $(25 \%)$ \\
DP & 25 & $(5 \%)$ & 138 & $(18 \%)$ \\
\hline Total & 493 & $(100 \%)$ & 1245 & $(40 \%)$ \\
\hline
\end{tabular}

*Porcentaje de artículos cientificos que aporta la revista al estudio. ** Porcentaje de artículos científicos sobre el total de titulos publicados por la revista. RPMESP: Revista Peruana de Medicina Experimental y Salud Pública. AFM: Anales de la Facultad de Medicina. RGP: R. de Gastroenterologia del Perú. RMH: R. Médica Herediana. AMP: Acta Médica Peruana. RPP: R. Peruana de Pediatria. DP: Dermatologia Peruana.

mero de revistas en las que ha publicado, y e) la colaboración más intensa.

La interacción de autores e instituciones se grafica a través de redes de colaboración, en las que se considera como 'nodo' al autor o institución que se relaciona con otros 'nodos' a través de 'líneas', las mismas que tienen una mayor intensidad conforme los nodos se relacionan más en diversos artículos; igualmente, los 'nodos' se diferencian en su tamaño y color dependiendo de las características del autor (cambia de tamaño de acuerdo al total de artículos) o institución (cambia de color de acuerdo al tipo de institución). Para este análisis, se ha identificado todas las combinaciones de pares de autores o instituciones en cada artículo. Para una mejor visualización solo se muestra los autores con más de cinco artículos y las instituciones con diez a más.

Se agrega además una tabla de características generales sobre autoría e instituciones por revista, en la que se describe el número de autores e instituciones por artículo según cada revista, y el porcentaje de artículos de la misma institución.

El cálculo de los indicadores bibliométricos, de redes sociales y la construcción de las representaciones gráficas de las redes de autores e instituciones, fue realizado utilizando los programas Excel 2007, el programa TextToPajek desarrollado en la Universidad Complutense de Madrid, y el programa de 
Tabla 3. Número de investigaciones publicadas y colaboración anual de autores e instituciones en revistas médicas peruanas.

\begin{tabular}{cccccccccc} 
Año & $\begin{array}{c}\mathrm{N}^{0} \\
\text { artículos }\end{array}$ & \multicolumn{2}{c}{$\begin{array}{c}\mathrm{N}^{0} \text { de artículos en } \\
\text { coautoría (\%) }\end{array}$} & $\mathrm{N}^{0}$ firmas & IFA & $\begin{array}{c}\mathrm{N}^{0} \\
\text { autores* }\end{array}$ & $\begin{array}{c}\mathrm{N}^{0} \text { autores } \\
\text { nuevos }\end{array}$ & $\begin{array}{c}\mathrm{N}^{0} \text { de artículos en } \\
\text { colaboración institucional (\%) }\end{array}$ \\
2005 & 107 & 94 & $(87,9 \%)$ & 434 & 4,1 & 387 & 387 & 67 & $(62,6 \%)$ \\
2006 & 122 & 113 & $(92,6 \%)$ & 529 & 4,3 & 457 & $372(81 \%)$ & 68 & $(55,7 \%)$ \\
2007 & 130 & 125 & $(96,2 \%)$ & 604 & 4,6 & 523 & $419(80 \%)$ & 73 & $(56,2 \%)$ \\
2008 & 134 & 120 & $(89,6 \%)$ & 564 & 4,2 & 501 & $362(72 \%)$ & 69 & $(51,5 \%)$ \\
Total & 493 & 452 & $(91,7 \%)$ & 2131 & 4,3 & 1540 & & 277 & $(56,2 \%)$ \\
\hline
\end{tabular}

IFA = Índice firmas por artículo.

* El total representa el número de autores nuevos en todos los años, por ello la suma no coincide.

** Se registra como autor nuevo al que haya publicado a partir del año de referencia.

análisis y visualización de redes PAJEK, mostrando los resultados con frecuencias, porcentajes, y los gráficos de redes descritos.

Como potenciales limitaciones metodológicas, se identificó autores que no señalaron su filiación institucional o que colocaban filiaciones institucionales diferentes en un mismo periodo de publicación; de igual modo existían errores ortográficos en la forma de escribir sus nombres o de su institución. En el caso de errores ortográficos de las instituciones, se corrigió al hacer la normalización de datos, pero no se normalizó las filiaciones institucionales de los autores en un mismo periodo ni se identificó la filiación de los autores que no la señalaban.

\section{RESULTADOS}

Se analizó 493 artículos que reunían 2131 firmas de 1540 autores, lo que sitúa el IFA en 4,3 para el periodo 2005 a 2008. El 91,7\% de los artículos fue realizado en coautoría, mientras $56,2 \%$ fue efectuado de manera conjunta por dos

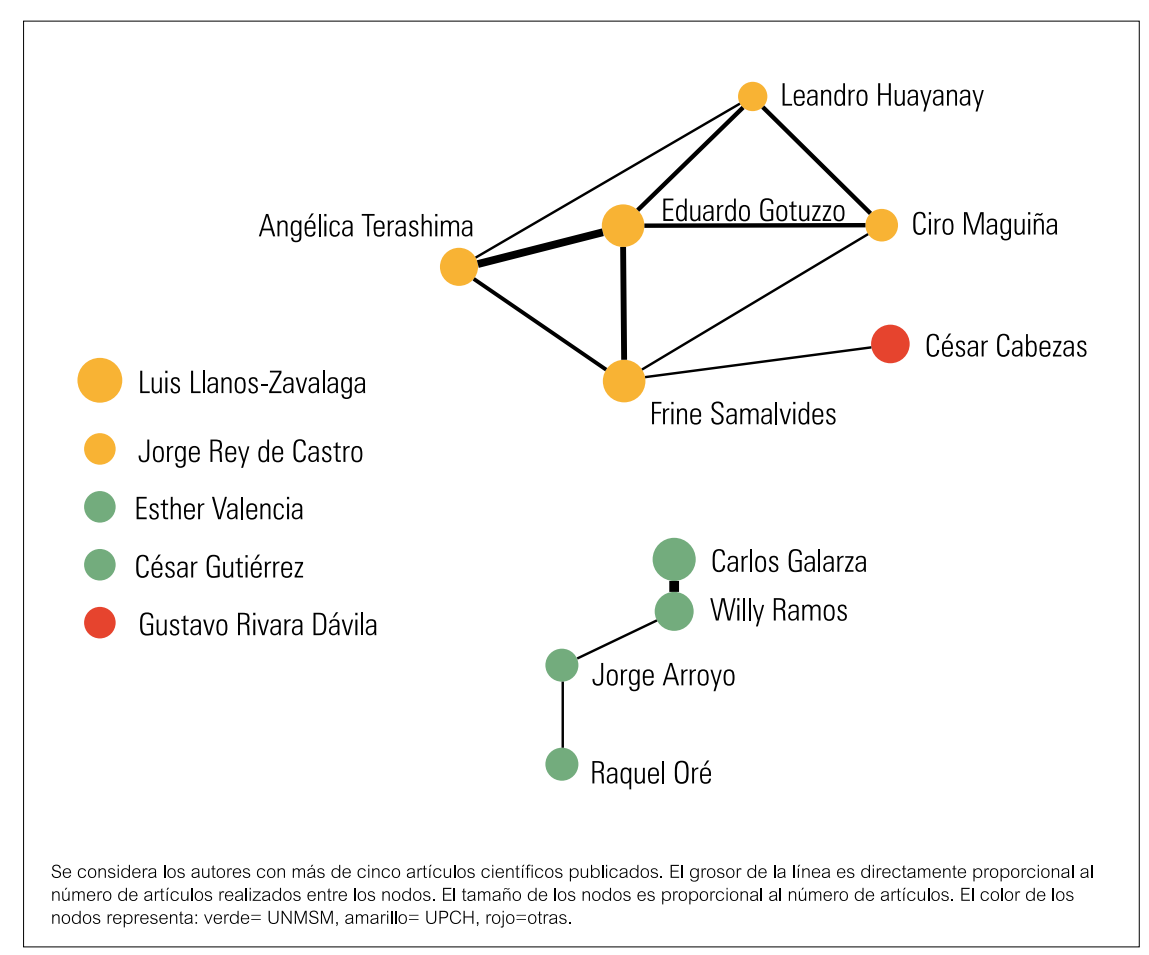

Figura 1. Redes de colaboración entre los autores más productivos en las revistas médicas peruanas. $2005-2008$.

instituciones. Se registró un promedio de 467 autores cada año; de ellos, un promedio de $385(77,9 \%)$ fueron autores nuevos. En la tabla 3 se recoge la evolución anual.

El 70,5\% de los investigadores tuvo solo un artículo registrado en el periodo de estudio, mientras $0,9 \%$ (15 autores) tuvo más de cinco artículos; de ellos, dos tuvieron de diez a más (considerados como grandes productores). Los patrones de colaboración de los primeros 15 autores son recogidos en la tabla 4; se registra además el número de revistas en las que han publicado, de firmas y colaboradores, y su relación con el número de artículos.

$\mathrm{Al}$ realizar el análisis de redes se observa que el principal grupo de investigadores de la UN Mayor de San Marcos tuvo una pobre interacción entre ellos y no estableció relaciones con el grupo de investigadores de la U Peruana Cayetano Heredia, siendo este un grupo mayor y con más interacción.

Se identificó a 271 instituciones; de ellas, 13 fueron grandes productores (con diez a más artículos); se recoge las características de su producción y colaboración en la tabla 5. La UN Mayor de San Marcos y la U Peruana Cayetano Heredia tuvieron cada una 138 y 125 artículos, habiendo colaborado con 67 y 75 instituciones distintas, respectivamente. Entre las instituciones más productivas, la UN Trujillo fue la que menos artículos en colaboración tuvo (45\%).

En el análisis de redes de colaboración, se aprecia que los principales 
Tabla 4. Autores más productivos y patrones de colaboración en revistas médicas peruanas. $2005-2008$.

\begin{tabular}{cccccccc} 
Autores & Artículos & N $^{0}$ Revistas & N $^{0}$ Firmas & IFA & N $^{0}$ CoAut* & $\begin{array}{c}\text { Colaboración } \\
\text { más intensa** }\end{array}$ & Aut/Art*** $^{* *}$ \\
Eduardo Gotuzzo & 11 & 4 & 61 & 5,5 & 46 & 4 & 4,2 \\
Luis Llanos Zavalaga & 10 & 1 & 25 & 2,5 & 17 & 5 & 1,7 \\
Frine Samalvides & 9 & 4 & 38 & 4,2 & 32 & 3 & 3,6 \\
Carlos Galarza & 9 & 3 & 67 & 7,4 & 39 & 6 & 4,3 \\
Willy Ramos & 8 & 4 & 66 & 8,3 & 37 & 6 & 4,6 \\
Angélica Terashima & 8 & 3 & 39 & 4,9 & 29 & 4 & 3,6 \\
César Cabezas & 8 & 1 & 38 & 4,8 & 35 & 3 & 4,4 \\
Ciro Maguiña & 7 & 2 & 39 & 5,6 & 36 & 2 & 5,1 \\
Jorge Arroyo & 7 & 2 & 42 & 6,0 & 28 & 5 & 4,0 \\
Raquel Oré & 7 & 1 & 39 & 5,6 & 26 & 3 & 3,7 \\
Esther Valencia & 6 & 3 & 45 & 7,5 & 27 & 4 & 4,5 \\
Leandro Huayanay & 6 & 3 & 36 & 6,0 & 34 & 2 & 5,7 \\
Jorge Rey de Castro & 6 & 3 & 16 & 2,7 & 8 & 5 & 1,3 \\
César Gutiérrez & 6 & 2 & 26 & 4,3 & 26 & 1 & 4,3 \\
Gustavo Rivara Dávila & 6 & 1 & 50 & 8,3 & 45 & 3 & 7,5 \\
\hline
\end{tabular}

IFA = Índice Firmas por Artículo. * Número de investigadores diferentes que han participado en el conjunto de los artículos que ha firmado cada uno de los autores. ${ }^{* *}$ Es el mayor número de artículos realizados con alguno de sus colaboradores. ${ }^{* * *}$ Índice que correlaciona el tamaño del grupo de trabajo de cada autor con el número de coautores y con su productividad.

nodos corresponden a las cuatro instituciones con mayor productividad; todas tienen algún grado de relación directa o indirecta entre ellos. También se aprecia que, a pesar que algunas instituciones tienen más artículos que otras, tienen a su vez menos colaboración; por ejemplo, en la figura 2, el HN Dos de Mayo tiene más artículos en colaboración con la UN Mayor de San Marcos que el HN Rebagliati, aunque en la tabla 5 se muestra que este último tiene más artículos publicados.
La colaboración institucional fue mayor dentro de una misma institución $(41,8 \%)$ o entre instituciones de una misma región (36,7\%); en 2007 se registró la mayor colaboración internacional $(8,5 \%)$. Las demás características están descritas en la tabla 6.

En la tabla 7 se recoge las características generales por cada revista; observamos que la R Médica Herediana tiene un menor número de autores por artículo y la mayor colaboración por parte de un mismo autor, lo que refiere que un conjunto menor de autores publica en la misma revista, siendo el $90 \%$ la producción de la misma institución.

\section{DISCUSIÓN}

El análisis de la producción científica médica nacional nos permitió identificar a centros núcleos de investigación que reunían no solo la mayor producción institucional, sino que eran

Tabla 5. Instituciones más productivas y características de colaboración en revistas médicas peruanas. 2005 - 2008.

\begin{tabular}{|c|c|c|c|c|c|c|}
\hline \multirow{2}{*}{$\begin{array}{l}\text { Filiación } \\
\text { UN Mayor de San Marcos }\end{array}$} & \multirow{2}{*}{$\begin{array}{c}N^{0} \text { de artículos } \\
138\end{array}$} & \multicolumn{2}{|c|}{$\begin{array}{l}\mathrm{N}^{0} \text { artículos en } \\
\text { colaboración }\end{array}$} & \multicolumn{2}{|c|}{$\begin{array}{l}N^{0} \text { de instituciones distintas } \\
\text { con las que ha colaborado * }\end{array}$} & \multirow{2}{*}{$\begin{array}{c}\text { Colaboración } \\
\text { más intensa } \\
20\end{array}$} \\
\hline & & 87 & $(63 \%)$ & 67 & $(0,5)$ & \\
\hline U Peruana Cayetano Heredia & 125 & 71 & $(57 \%)$ & 75 & $(0,6)$ & 9 \\
\hline IN Salud & 70 & 54 & $(77 \%)$ & 56 & $(0,8)$ & 20 \\
\hline HN Edgardo Rebagliati Martins & 26 & 13 & $(50 \%)$ & 15 & $(0,6)$ & 4 \\
\hline HN Dos de Mayo & 25 & 17 & $(68 \%)$ & 20 & $(0,8)$ & 11 \\
\hline IN Salud del Niño & 20 & 18 & $(90 \%)$ & 18 & $(1,0)$ & 7 \\
\hline UN Federico Villarreal & 17 & 16 & $(94 \%)$ & 16 & $(1,0)$ & 5 \\
\hline H Arzobispo Loayza & 17 & 15 & $(88 \%)$ & 11 & $(0,7)$ & 6 \\
\hline H Guillermo Almenara Irigoyen & 14 & 11 & $(79 \%)$ & 14 & $(1,1)$ & 6 \\
\hline U San Martín Porres & 12 & 11 & $(92 \%)$ & 13 & $(1,2)$ & 6 \\
\hline UN Trujillo & 11 & 5 & $(45 \%)$ & 10 & $(1,0)$ & 2 \\
\hline IN Enfermedades Neoplásicas & 11 & 6 & $(55 \%)$ & 6 & $(0,6)$ & 2 \\
\hline HN Daniel A Carrión & 10 & 7 & $(70 \%)$ & 11 & $(1,2)$ & 4 \\
\hline
\end{tabular}

* Entre paréntesis figura el indice de colaboradores por articulo. U= Universidad, I= Instituto, $\mathrm{H}=$ Hospital, $\mathrm{N}=\mathrm{Nacional}$. 
Tabla 6. Número de colaboraciones institucionales agrupadas por tipo de colaboración y número total de artículos en colaboración en revistas médicas peruanas. $2005-2008$.

\begin{tabular}{|c|c|c|c|c|c|c|c|c|c|c|}
\hline \multirow{3}{*}{$\begin{array}{l}\text { Tipo de colaboración* } \\
\text { Colaboración intrainstitucional }\end{array}$} & \multicolumn{8}{|c|}{$\mathrm{N}^{0}$ de artículos en colaboración } & \multirow{2}{*}{\multicolumn{2}{|c|}{ Total }} \\
\hline & \multicolumn{2}{|c|}{ Año 2005} & \multicolumn{2}{|c|}{ Año 2006} & \multicolumn{2}{|c|}{ Año 2007} & \multicolumn{2}{|c|}{ Año 2008} & & \\
\hline & 37 & $(34,6 \%)$ & 52 & $(43,3 \%)$ & 53 & $(40,8 \%)$ & 63 & $(47, \%)$ & 205 & $(41,8 \%)$ \\
\hline Colaboración interinstitucional: & & & & & & & & & & \\
\hline - Misma región & 43 & $(40,2 \%)$ & 47 & $(39,2 \%)$ & 49 & $(37,7 \%)$ & 41 & $(30,6 \%)$ & 180 & $(36.7 \%)$ \\
\hline - Diferentes regiones & 12 & $(11,2 \%)$ & 11 & $(9,2 \%)$ & 10 & $(7,7 \%)$ & 16 & $(11,9 \%)$ & 49 & $(10.0 \%)$ \\
\hline Colaboración internacional & 9 & $(8,4 \%)$ & 4 & $(3,3 \%)$ & 11 & $(8,5 \%)$ & 9 & $(6,7 \%)$ & 33 & $(6,7 \%)$ \\
\hline Internacionales & 6 & $(5,6 \%)$ & 6 & $(5,0 \%)$ & 7 & $(5,4 \%)$ & 5 & $(3,7 \%)$ & 24 & $(4,9 \%)$ \\
\hline Total & 107 & & 120 & & 130 & & 134 & & 491 & \\
\hline
\end{tabular}

* No todos los artículos refieren una filiación institucional; por ello existe una diferencia con el total de artículos publicados.

núcleos de red y, por tanto, promovían la investigación con otras instituciones. Del mismo modo, reúnen a los investigadores más productivos, aunque con un nivel bajo de interrelación entre ellos. A su vez, estas instituciones poseían fuentes directas para difundir sus investigaciones a través de revistas institucionales, logrando así consolidar un espacio en los primeros puestos de producción científica nacional.
Los indicadores mostrados permiten una comparación con investigaciones internacionales ${ }^{(10,11)}$, pero están dirigidos a evaluar fenómenos generales, y no deberían ser considerados como indicadores absolutos; es más, no deben servir para valorar a personas o instituciones, sino ser utilizados para un análisis integral ${ }^{(2,11,12)}$. Por ejemplo, si solo valoramos la productividad de un investigador como el número de ar-

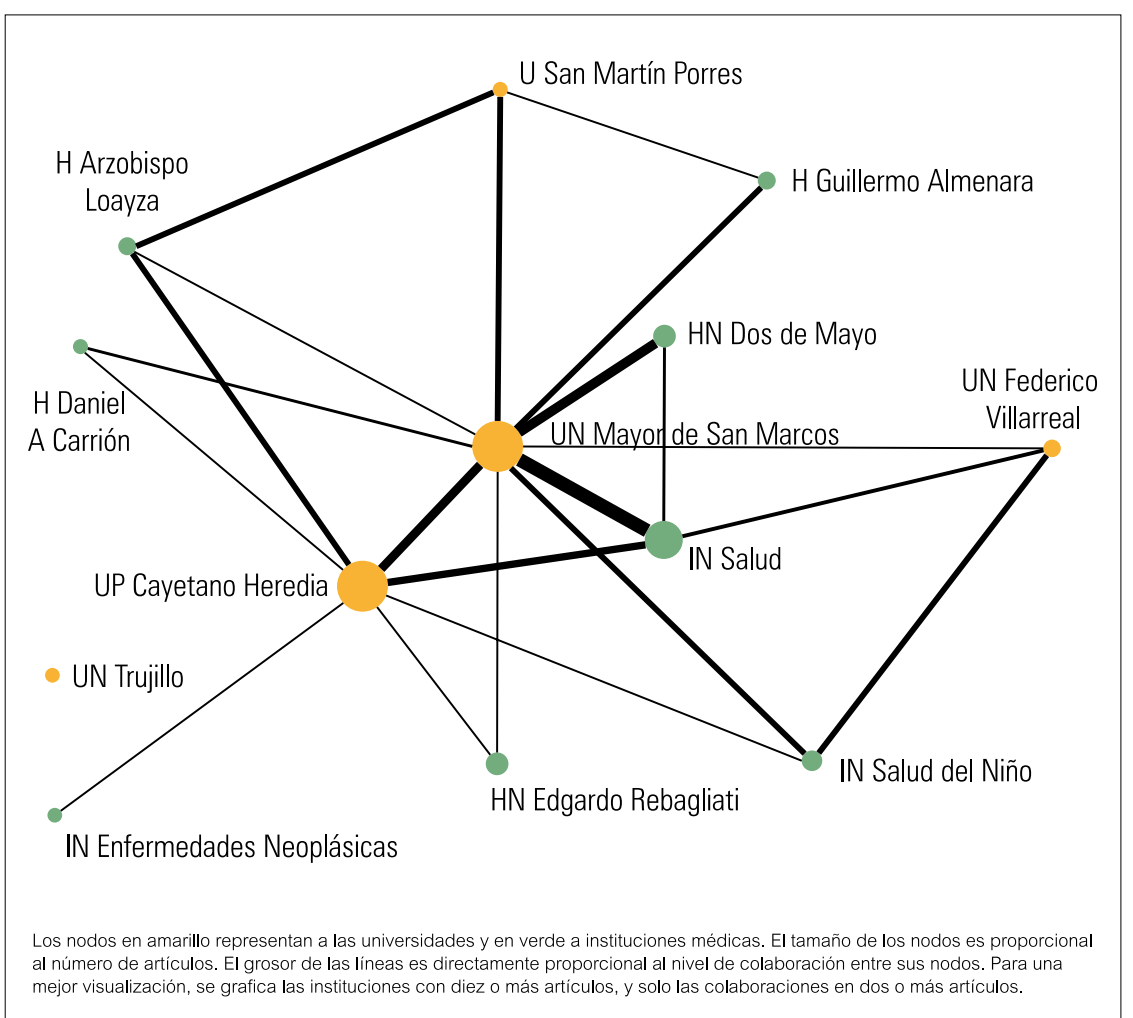

Figura 2. Redes de colaboración entre las instituciones más productivas en las revistas médicas peruanas, $2005-2008$. tículos publicados podríamos descuidar a aquellos que tienen publicaciones en una mayor variedad de revistas, por la obvia razón que al publicar en un mayor número de revistas, sus manuscritos serían evaluados por más investigadores ${ }^{(11)}$. Igualmente, índices de firmas más elevados pueden señalar una mayor apertura para investigar con diversos grupos, pero también pueden reflejar la 'utilidad' del investigador para facilitar publicaciones a grupos menores, o la 'disponibilidad' de permitir a otros grupos colocar su nombre como autor $^{(12,13)}$.

Considerando estas salvedades, los indicadores mostrados permiten evaluar mejor a instituciones y, como se aprecia en la tabla 5, podemos sugerir que las instituciones más productivas necesitan de una mayor colaboración para realizar sus investigaciones. Esta situación ya ha sido vista al analizar las publicaciones peruanas en revistas de alto impacto ${ }^{(3)}$, donde el porcentaje de colaboración se da principalmente con universidades provenientes de países de habla inglesa, posibles por convenios de colaboración institucionales y facilitados por los investigadores que en su momento viajaron para recibir formación de posgrado. En el contexto nacional, las colaboraciones no se dan necesariamente a través de convenios, sino por parte de la doble filiación que tienen los investigadores, quienes al formar parte de ambas instituciones permiten la interacción y reúnen además a otros investigadores. 
Tabla 7. Características generales sobre autoría e instituciones por revista.

$\begin{array}{cccccccc} & \text { RPMESP } & \text { AFM } & \text { RMH } & \text { RGP } & \text { DP } & \text { AMP } & \text { RPP } \\ \text { Artículos } & 139 & 106 & 72 & 74 & 25 & 42 & 35 \\ \text { No de autores } & 508 & 320 & 183 & 272 & 72 & 174 & 134 \\ \text { Índice Aut/Art } & 3,7 & 3,0 & 2,5 & 3,7 & 2,9 & 4,1 & 3,8 \\ \text { Mayor colaboración } & 8 & 7 & 10 & 5 & 6 & 4 & 6 \\ \text { No instituciones } & 134 & 60 & 32 & 49 & 24 & 54 & 27 \\ \text { Índice Ins/Art } & 1,0 & 0,6 & 0,4 & 0,7 & 1,0 & 1,3 & 0,8 \\ \text { Artículos de la misma institución } & 63(45 \%) & 76(72 \%) & 65(90 \%) & - & - & - & -\end{array}$

RPMESP: Revista Peruana de Medicina Experimental y Salud Pública. AFM: Anales de la Facultad de Medicina. RMH: R. Médica Herediana. RGP: R. de Gastroenterología del Perú. DP: Dermatologia Peruana. AMP: Acta Médica Peruana. RPP: R. Peruana de Pediatria. Nota: Se considera solo los artículos incluidos en el estudio. La mayor colaboración corresponde al autor que tiene más artículos en la revista. Solo se analizó el número de artículos de una misma institución para las revistas RPMESP, AFM y RMH, por tener una institución definida (IN de Salud, UN Mayor de San Marcos y U Peruana Cayetano Heredia respectivamente).

La colaboración institucional es mayor en las publicaciones en revistas de alto impacto ${ }^{(3)}$, que se puede deber gracias a la mayor calidad de los trabajos en colaboración y por el dominio del inglés. Sobre la mayor calidad de los trabajos, esta guarda relación con la importancia de la colaboración, que radica en la posibilidad de abordar problemas multidisciplinarios y complementar deficiencias ${ }^{(12-14)}$, tanto económicas, logísticas, de procedimientos, entre otros; por ello y con frecuencia, se ve que las instituciones más productivas no son 'competidoras autistas', sino que guardan fuertes relaciones entre ellas. Tal es el caso de la UN Mayor de San Marcos y la U Peruana Cayetano Heredia, que muestran una gran interrelación en la figura 2. Mientras otras, quizá deban gran parte de su producción precisamente a las redes de colaboración, presentando colaboraciones a veces tan grandes como su misma producción.

En este análisis vemos a cinco universidades peruanas en la red de principales productores, mientras en la red de publicación en revistas extranjeras solo figuran la UN Mayor de San Marcos y la U Peruana Cayetano Heredia ${ }^{(3)}$. Preocupa ver que la participación universitaria es menor a la que debería esperarse, pues si entendemos a las universidades como responsables de brindar y generar conocimiento, el rol que realmente cumplen no es tal; a diferencia de las universidades extranjeras que colaboran con nosotros.

Es pertinente señalar también, que el dominio del idioma inglés facilita la publicación de artículos en revistas extranjeras y de alto impacto. Esto se puede sugerir al ver que el $98 \%$ de la publicación peruana en revistas de alto impacto está en idioma inglés, donde la colaboración con instituciones de habla inglesa es mayor al 60\% ${ }^{(3)}$, en comparación al casi $7 \%$ de colaboración extranjera en publicaciones en revistas peruanas. Aunque es un porcentaje bajo, llama la atención y puede significar que los autores extranjeros consideran que nuestras revistas, a pesar de sus limitaciones, son espacios idóneos para la comunicación de sus investigaciones, brindándoles así un reconocimiento indirecto; o al contrario, son fuentes de fácil publicación para aquellos autores extranjeros que no pueden ver sus artículos publicados en revistas de impacto internacional $^{(11)}$.

Del mismo modo, al analizar las características generales de las revistas, se aprecia que hubo en promedio 3,6 autores por artículo, que es un valor razonable, pues IFA elevados no señalan necesariamente una mayor colaboración para obtener un mejor producto, sino quizá la aparición de autorías ficticias. Sin embargo, es importante señalar que cada vez son más las investigaciones multidisciplinarias, que ameritan por tanto una mayor colaboración de autores ${ }^{(8)}$.

Con los análisis realizados no se puede juzgar la intervención de estas instituciones en la investigación, vale decir, qué tanto aportó la institución con el autor (proporcionó insumos, equipos, infraestructura, o solo la mención de pertenecer a dicha institución).

Como potenciales limitaciones del estudio, figura en primer lugar los errores en los datos de filiación, con lo que podría duplicarse autores en algunos casos. También, se seleccionó un conjunto de revistas que no representa toda la producción biomédica nacional, a pesar que se escogió las revistas con mayor visibilidad, ya que debemos agregarle la publicación en revistas extranjeras y otras oriundas de menor difusión. Tampoco se consideró la literatura publicada en revistas extranjeras; ni artículos que no tienen la misma estructura que los artículos científicos, pero que desarrollan investigaciones en otras áreas temáticas.

Dado que las universidades e institutos de investigación son los responsables de la producción de conocimiento, recomendamos que incentiven a sus vicerrectorados o departamentos de investigación a incrementar su pro- 
ducción científica, brindándoles los insumos y presupuestos necesarios para las investigaciones ${ }^{(15)}$. En ese sentido, la gestión en ciencia, tecnología e innovación es un área interesante que ha sido incluida por instituciones de vanguardia en investigación ${ }^{(16)}$, generando incluso una política nacional de investigación, por lo que se recomienda fortalecer o fomentar el desarrollo de estos sistemas nacionales de investigación de salud, como encargados de la promoción de las actividades en cooperación.

Finalmente, el presente estudio marca solo un corte, a partir del cual podemos comparar, analizar y discutir nuestro actual estado en investigación, necesitando por ello generar los sistemas para poder repetir, sin tantas limitaciones como las ya expuestas, estudios similares y dedicados a cada aspecto involucrado.

En conclusión, la investigación médica nacional se desarrolla a través de pocos núcleos de investigación, principalmente universitarios, que están centralizados, y con una apertura moderada a otros nodos en desarrollo. Con ello, podemos recomendar que se incentive a demás instituciones involucradas en la investigación, llámense centros médicos o entidades educadoras, a la promoción de la investigación a través de la colaboración directa con ellas, logrando así incrementar nuestras redes de trabajo y potenciar sus equipos de investigación.

\section{REFERENCIAS BIBLIOGRÁFICAS}

1. Pamo O. Estado actual de las publicaciones periódicas científicas médicas del Perú. Rev Med Hered. 2005;16(1):65-73.

2. López-Piñero JM, Terrada ML. Los indicadores bibliométricos y la evaluación de la actividad médicocientífica. (I) Usos y abusos de la bibliometria. Med Clin (Barc). 1992;98:64-8.

3. Huamani $C$, Mayta-Tristán P. Producción científica peruana en medicina y redes de colaboración, análisis del Science Citation Index 2000-2009. Rev Per Med Exp Salud Pública. 2010;27 (3):314-24.

4. Boscha X, Villacastín JP, Alfonso F. Difusión, reconocimiento científico y repercusión internacional. Rev Esp Cardiol. 2001;54:1463-5.

5. González de Dios J, Sempere AP, AleixandreBenavent R. Las publicaciones biomédicas en España a debate (II): las 'revoluciones' pendientes y su aplicación a las revistas neurológicas. Rev Neurol. 2007;44:101-12.

6. Álvarez-Solar M, López-González ML, Cueto-Espinar A. Indicadores bibliométricos, análisis temático y metodológico de la investigación publicada en España sobre epidemiología y salud pública (19881992). Med Clin (Barc). 1998;111:529-35.

7. López-Piñero JM, Terrada ML. El consumo de información científica nacional y extranjera en las revistas médicas españolas: un nuevo repertorio destinado a su estudio. Med Clin (Barc). 1994;102:104-12

8. López-Piñero JM, Terrada ML. Los indicadores bibliométricos y la evaluación de la actividad médico-científica. (III) Los indicadores de producción, circulación y dispersión, consumo de la información y repercusión. Med Clin (Barc). 1992;98:142-8.

9. Huamani C, Pacheco-Romero J. Visibilidad y Producción de las Revistas Biomédicas Peruanas. Rev Gastroenterol Perú. 2009;29(2):132-9.

10. González-Alcaidea G, Alonso-Arroyo A, Gonzáles de Dios J, Sempere AP, Valderrama-Zuriána JC, Aleixandre-Benaventa R. Redes de coautorías y colaboración institucional en Revista de Neurología. Rev Neurol. 2008;46(11):642-51.

11. Estrada Lorenzo JM, Villar-Álvarez F, Pérez-Andrés C, Rebollo-Rodríguez MJ. Estudio bibliométrico de los artículos originales de la Revista Española de Salud Pública (1991-2000). Parte segunda: productividad de los autores y procedencia institucional y geográfica. Rev Esp Salud Pública. 2003;77:333-46.
12. Pérez-Andrés C, Estrada-Lorenzo JM, Villar-Álvarez F, Rebollo-Rodríguez MJ. Estudio bibliométrico de los artículos originales de la Revista Española de Salud Pública (1991-2000). Parte primera: Indicadores generales. Rev Esp Salud Pública. 2002;76:659-72.

13. Valderrama-Zuriána JC, González-Alcaidea G, Valderrama-Zurián FJ, Aleixandre-Benaventa $R$, Miguel-Dasit A. Redes de coautorias y colaboración institucional en Revista Española de Cardiología. Rev Esp Cardiol. 2007;60(2):117-30.

14. Wagner CS, Leydesdorff L. Network structure, self-organization, and the growth of international collaboration in Science. Research Policy. 2005;34:1608-18.

15. Gotuzzo E, González E, Verdonck K. Formación de investigadores en el contexto de proyectos colaborativos: experiencias en el Instituto de Medicina Tropical "Alexander von Humboldt", Universidad Peruana Cayetano Heredia. Rev Peru Med Exp Salud Publica. 2010;27(3):419-27.

16. Yagui M, Espinoza M, Caballero P, Castilla T, Garro $G$, Yamaguchi LP, et al. Avances y retos en la construcción del sistema nacional de investigación en salud en el Perú. Rev Peru Med Exp Salud Publica. 2010;27(3):387-97.

Artículo recibido el 28 de agosto de 2011 y aceptado para publicación el 14 de octubre de 2011.

Este estudio forma parte de la tesis para optar por el titulo de médico cirujano, titulada "Análisis de la investigación cientifica en revistas médicas peruanas, $2005-2008$ ".

\section{Agradecimientos:}

Al Dr. Gregorio Gonzáles Alcaide, del Departament d'Història de la Ciència i Documentació, Universitat de València, España; por su asesoramiento en el diseño y análisis de redes de colaboración.

\section{Correspondencia:}

Charles Huamani Saldaña

Av. Arriba Perú 1154, Villa el Salvador. Lima 42, Perú

Teléfono: (+51-1) 9928-14710/287-1397

Correo electrónico: huamani_ca@hotmail.com. 